\title{
Programa de estudios para una arquitectura sostenible y sensible al patrimonio
}

\author{
Pedro Paulo Palazzo | Universidad de Brasilia \\ URL de la contribución <www.iaph.es/revistaph/index.php/revistaph/article/view/4983>
}

\section{Principios}

Se proclama a menudo que el desarrollo sostenible, la conservación del patrimonio y la práctica corriente de la construcción son aspectos de una misma disciplina integrada de la arquitectura. La misma existencia de cierto edificio histórico implica ahorro energético y captura de carbono mucho más grandes que la más eficiente nueva construcción (Elefante 2012). Sin embargo, las capacidades técnicas para dar mantenimiento a las estructuras tradicionales suelen ser muy distintas de la tecnología industrial asociada al desarrollo sostenible. Estas diferencias no son disparidades superficiales entre objetivos convergentes, sino que muestran una incompatibilidad de principios entre determinada cultura de patrimonio a la que interesa lo existente, lo regional y lo artesanal, y la cultura del desarrollo industrial dedicada a producir y desechar siempre más, aunque produzca y deseche productos con la etiqueta "ecológica".

Desde luego, la crisis provocada por la construcción industrializada no es solo climática y cultural, sino sobre todo económica y social. Además de los resultados ambientales que advienen de las propiedades intrínsecas de los materiales, la construcción tradicional favorece un proceso productivo sostenible en otros aspectos (Vellinga 2006).

Una de las más críticas consecuencias del cambio climático es el desplazamiento de procesos productivos que se organizan en cadenas globales y la consecuente privación económica de comunidades locales (Hallegatte, Fay y Barbier 2018). La construcción tradicional y la conservación de edificios históricos no solo contribuyen a la lucha directa contra el cambio climático, como también refuerzan cadenas productivas en escala local y regional (Robertson 2012).

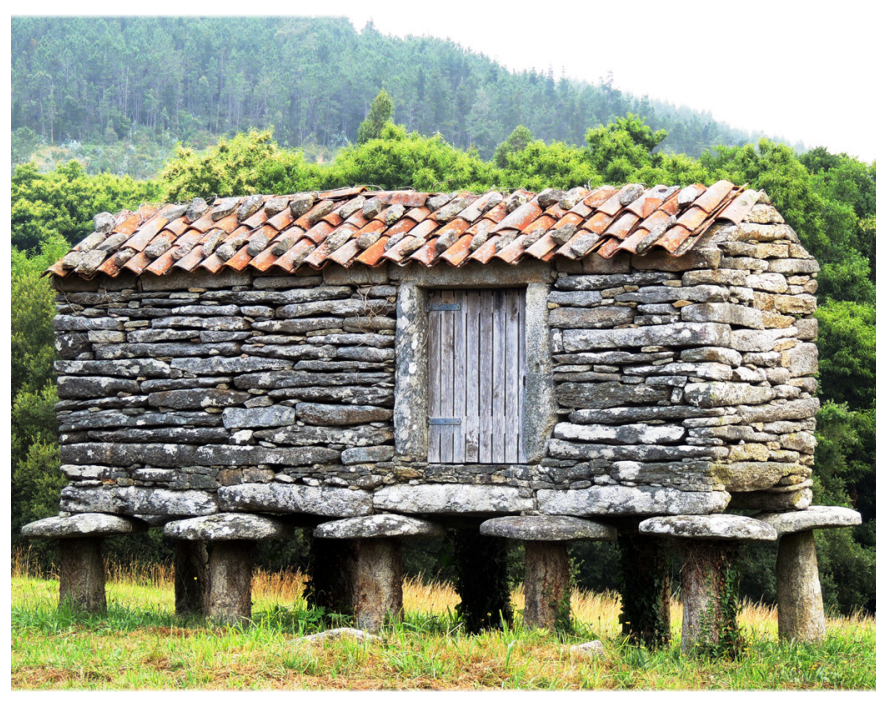

Hórreo en el Pazo do Allo en Zas (Coruña) | foto Jose Luis Cernadas Iglesias

Es cierto que, para solucionar la crisis climática, habrá que actuar sobre las estructuras productivas del mercado inmobiliario, pero para que cambien las estructuras debe existir aptitud técnica alternativa en la construcción y en su regulación. Por eso, la formación de arquitectos tiene un papel central en este proceso. La aptitud para emplear de modo corriente materiales de baja energía incorporada (Röck et ál. 2020) y para hacerlo en armonía con el carácter de las construcciones vernáculas será capaz de cerrar la brecha conceptual entre la conservación de edificios de valor patrimonial y las metas de reducción de emisiones de carbono en la industria de la construcción. Para capturar localmente las ganancias socioeconómicas que puede generar la conservación de la construcción tradicional como materia y como práctica, se necesita ubicar la enseñanza de los métodos tradicionales de construcción con materiales regionales en el centro del programa de estudios en arquitectura. 
El programa de estudios que planteo a continuación privilegia desde luego la materialidad de la construcción como punto de partida, más que la noción de diseño puramente formal y abstracto que domina la didáctica de arquitectura hoy día. Este orden de prioridades ya fue una vez normal en las escuelas de bellas artes (Curtis 1923; Guadet 1901). El programa se podrá adaptar a un sistema universitario, o incluso ser ofrecido como educación continuada para profesionales. El hilo conductor del programa es mostrar cómo la apariencia de "ineficiencia" de la construcción tradicional resulta en realidad más resiliente a largo plazo que la "eficiencia" de los cálculos, productos y métodos de la arquitectura industrializada convencional.

\section{Programa de estudios. Propuesta}

Se estructuraría en tres ciclos:

\section{El primer ciclo dedicado a los elementos:}

> Los elementos de construcción: los materiales naturales y elementos que pertenecen a la tradición constructiva de cada región (Asquith y Vellinga 2006); las estructuras eficientes no son resilientes; fundaciones, tapias, estructuras trabeadas y arqueadas, cubiertas y revestimientos; y priorizar la mantenencia local sobre el reemplazo (Thomsen y Van der Flier 2011).

> Los elementos de composición: las respuestas holísticas al clima regional (Correia, Gilberto y Rocha 2014): ventilación natural y gestión de la humedad, los riesgos de la impermeabilización y de la estanqueidad para la salud humana y la durabilidad de los edificios; el carácter de las plantas bajas como expresión de propósitos sociales básicos (Westfall 1991); y la composición de casas y del espacio público con volúmenes de formas definidas (Krier 1988).

> Hacer arquitectura tradicional: generar "estructuras vivas" (Alexander 2002; Cogdell 2019) en vez de diseñar conceptos "de autor"; el desarrollo incremental de la forma (Bacon 1974); las cuatro operaciones vitruvianas: ordenar, articular, apropiar y proporcionar (Scranton 1974).
2. El segundo, a las prácticas:

> Tipología y proyecto: la intervención en el ambiente construido es un proceso y no forma estática (Caniggia y Maffei 1987); contra el concepto de "diseño" abstracto, la "composición" como manipulación de tipos y espacios con sentido cultural (Amoruso 2018).

> Estilo y otras convenciones culturales: Ios "estilos" de la arquitectura son respuestas holísticas y resilientes a retos constructivos, climáticos y culturales, y hace falta respetarlos por eso. Se exploraría el continuo entre la arquitectura vernácula y la erudita (Krier 2009) y la analogía formal y técnica en el patrimonio histórico (Capitel 1988).

$>$ Vegetación y agua: la gestión del paisaje urbano y rural es el reto de la arquitectura (Muratori 1967; Upton 1991); también la adaptación al clima y soluciones regionales de manejo del agua y de la vegetación.

> Urbanización: la interdependencia entre la subdivisión del espacio urbano y la tipología arquitectónica (Corona Martínez 2009; Panerai 2012); romper el paradigma tecnocrático de megaproyectos públicos o privados en favor de una urbanización gradual y descentralizada; y el proceso tipológico en la escala del territorio (Caniggia 1997).

\section{El tercer ciclo abordaría problemas complejos:}

Es decir, retos específicos a cada región y sus problemas climáticos, socioeconómicos y culturales, deteniéndose en el pensamiento crítico de las normas y los instrumentos de gestión (Tafuri 1980) que promueven u obstan la construcción tradicional y la conservación del patrimonio. 


\section{BIBLIOGRAFÍA}

- Alexander, C. (2002) The Nature of Order. Berkeley, California: Center for Environmental Structure

- Amoruso, G. (2018) Rappresentare la resilienza: modelli conoscitivi per la ricostruzione. Milano: Lampi di Stampa. Disponible en: https://re.public.polimi.it/handle/11311/1095492 [Consulta: 14/09/2021]

- Asquith, L. y Vellinga, M. (ed.) (2006) Vernacular Architecture in the Twenty-First Century: Theory, Education and Practice. London; New York: Taylor \& Francis

- Bacon, E.N. (1974) Design of Cities. New York: Penguin

- Caniggia, G. (1997) Ragionamenti di tipologia: operatività della tipologia processuale in architettura. Firenze: Alinea

- Caniggia, G. y Maffei, G.L. (1987) I/ progetto nell'edilizia di base: composizione architettonica e tipologia edilizia: 2. Biblioteca di Architettura e Urbanistica. Venezia: Marsilio

- Capitel, A. (1988) Metamorfosis de monumentos y teorías de la restauración. Madrid: Alianza

- Cogdell, C. (2019) Toward a Living Architecture? Complexism and Biology in Generative Design. Minneapolis: University of Minnesota Press. Disponible en: https://doi.org/10.5749/j. ctv9b2tnw [Consulta: 13/07/2021]

- Corona Martínez, A. (2009) Ensayo sobre el proyecto. Buenos Aires: Nobuko

- Correia, M.; Gilberto,C. y Rocha, R. (ed.) (2014) Vernacular Heritage and Earthen Architecture: Contributions for Sustainable Development; Proceedings of CIAV 2013, 7th ATP, Versus, Vila Nova de Cerveira, Portugal, 16-20 October 2013. Leiden: CRC Press

- Curtis, N.C. (1923) Architectural Composition. Cleveland, Ohio: J. H. Janson

- Elefante, C. (2012) The Greenest Building Is... One That Is Already Built. Forum Journal, vol. 27, n. ${ }^{\circ} 1$, pp. 62-72. Disponible en: https://muse.jhu.edu/article/494514 [Consulta: 12/07/2021]

- Guadet, J. (1901) Éléments et théorie de l'architecture. Paris: Aulanier

- Hallegatte, S., Fay, M. y Barbier, E.B. (2018) Poverty and Climate Change: Introduction. Environment and Development Economics, vol. 23, n. ${ }^{\circ}$ 3, pp. 217-33. Disponible en: https://doi.org/10.1017/S1355770X18000141 [Consulta: 12/07/2021]

- Krier, L. (2009) The Architecture of Community. Washington, D.C.: Island Press (Thadani, D.A. y Hetzel, P.J. edicion)

- Krier, R. (1988) Architectural Composition. New York: Rizzoli
- Muratori, S. (1967) Civiltà e territorio. Roma: Centro studi di storia urbanistica

- Panerai, P. (2012) Analyse urbaine. Marseille: Parenthèses

- Robertson, M. (ed.) (2012) Sustainable Cities: Local Solutions in the Global South. Rugby, Warwickshire: Practical Action

- Röck, M., Saade, M.R.M., Balouktsi, M., Rasmussen, F.N., Birgisdottir, H., Frischknecht, R., Habert, G., Lützkendorf, T. y Passer, A. (2020) Embodied GHG Emissions of BuildingsThe Hidden Challenge for Effective Climate Change Mitigation. Applied Energy, vol. 258, 114107. Disponible en: https://doi. org/10.1016/j.apenergy.2019.114107 [Consulta: 12/07/2021]

- Scranton, R.L. (1974) Vitruvius' Arts of Architecture. Hesperia: The Journal of the American School of Classical Studies at Athens, vol. 43, n. ${ }^{\circ} 4$, pp. 494-99. Disponible en: https://doi.org/10.2307/147496 [Consulta: 12/07/2021]

- Tafuri, M. (1980) La Sfera e il labirinto: avanguardie e architettura da Piranesi agli anni '70. Torino: G. Einaudi

- Thomsen, A. y Van der Flier, K. (2011) Understanding obsolescence: a conceptual model for buildings. Building Research \& Information, vol. 39, n. ${ }^{\circ} 4$, pp. 352-62. Disponible en: https://doi.org/10.1080/09613218.2011.576328 [Consulta: 12/07/2021]

- Upton, D. (1991) Architectural History or Landscape History? Journal of Architectural Education, vol. 44, n. ${ }^{\circ} 4$, pp. 195-99. Disponible en: https://doi.org/10.2307/1425140 [Consulta: 12/07/2021]

- Vellinga, M. (2006) The Inventiveness of Tradition: Vernacular Architecture and the Future. Perspectives in Vernacular Architecture, vol. 13, n. ${ }^{\circ}$ 2, pp. 115-28. Disponible en: https:// www.jstor.org/stable/20355388 [Consulta: 12/07/2021]

- Westfall, C.W. (1991) Building Types. En: Westfall, C.W. y Jan van Pelt, R. (ed.) Architectural Principles in the Age of Historicism, 138-67. New Haven: Yale University Press 